density wave (a quantity under debate at present), the frequency of the noise can be accounted for ${ }^{14}$.

Recently there was a report of dark-field electron microscopy on $\mathrm{NbSe}_{3}$ (ref. 15). An image of an area of the sample is formed using the superlattice reflection alone; thus any feature in the imaging is due to the charge density wave formation. It is found that stripes approximately $200 \AA$ wide run along the conducting axis, and across the stripes are bands of lighter and darker intensities, suggestive of a Moiré pattern. It appears that the charge density wave structure may itself be broken into domains and that more than one superlattice wave vector is possible, so that interference between them forms the Moiré pattern. What is even more intriguing is that at low temperatures the bands are found to shift in time, so that the entire pattern shimmers on a time scale of a fraction of a second. This suggests some time-dependent motion of the charge density wave, but it is occurring on such a slow time scale that its connection with the nonlinear and frequency-dependent conductivity is unclear.

It is rare to find a material like $\mathrm{NbSe}_{3}$ that exhibits such a rich variety of unusual properties. At present the idea of a sliding charge density wave provides a framework for the qualitative understanding of many of these properties, but clearly much work remains to be done before a quantitative understanding is achieved.

\title{
Mobile genetic elements: new Soviet studies
}

\section{from A. D. Mirzabekov}

A CONFERENCE devoted to studies of eukaryotic mobile dispersed genetic elements was held on November 14, 1980 as a session of the seminar 'Chromosoma' at the Institute of Molecular Biology (USSR Academy of Sciences, Moscow).

In the Drosophila melanogaster genome mobile dispersed genetic (mdg) elements are multiple, scattered throughout the whole genome, and can move around. They are also actively transcribed (G.P. Georgiev*). At present, about 20 families of mdg elements have been described and comprise as much as five per cent of the whole $D$. melanogaster genome. The variable location of mdg elements is the most striking example known of genomic differences between individuals of the same species.

Y.V. Ilyin' described the structural organization of the two elements, mdgl $(\mathrm{a} \sim 7 \mathrm{kilobase}(\mathrm{kb})$ element including the previously described Dm225 and Dm234 sequences) and mdg3 ( $\sim 5 \mathrm{~kb})$ cloned in the plasmid pBR322. In cell culture, $\operatorname{mdg} 1$ and mdg 3 are amplified but not tandemly repeated. Like all other transposons, mdg1 and mdg 3 contain long terminal repeats (LTRs) at the ends of the gene.

Sequence data show the structure of the LTRs of mdg3 (Bayev et al. Nucleic Acids Res. 8; 3263, 1980; A.S Krayer, V. Kulguskin*) to be perfect direct repeats 268 bp long flanked by two mismatched 18 bp inverted repeats. The complete mdg 3 element is then bounded by short ( $5 \mathrm{bp}$ ) direct repeats probably derived from host target sequence duplication. Mdg1 contains longer perfect direct repeats (442 bp) flanked by mismatched 16 bp inverted

* Institute of Molecular Biology, Moscow. tInstitute of Molecular Genetics, Moscow. repeats. In this respect, the organization of mdg1 LTR is similar to LTRs of the copia gene (Levis et al. Cell 21; 580, 1980) or terminal repeats of integrated proretroviruses (Beveren et al. Proc. natn. Acad. Sci. U.S.A. 77; 3307, 1980; Dhar et al. ibid; 3937, 1980). Within both LTRs, signal sequences for transcription initiation (Hogness box) and polyadenylation (AATAAA) are found.

The whole mdg sequences of $\mathrm{mdgl}$ and mdg 3 are transcribed, initiation and termination sites being localized within LTRs (V.G. Chmeliauskaite*). Both DNA strands are transcribed giving rise to the long double-stranded RNA stretches found in total poly(A) ${ }^{+}$RNA from cell culture. However, the efficiency of transcription of one strand is much higher than that of the other. At least in $D$. melanogaster, hybridization to very long double-stranded RNA can be used as a diagnostic test in the search for new mdg elements. Besides full-length transcripts, spliced poly $(\mathrm{A})^{+}$RNAs were found among mdg1- and mdg3-specific RNA sequences. The major part of mdg transcripts are located in free ribonucleoprotein particles in the cytoplasm and only a minor fraction is bound to polysomes.

M.D. Golubovsky (Institute of Cytology and Genetics, Novosibirsk) showed that the frequency of an unstable mutation in the $s n$ locus of $D$. melanogaster can simultaneously increase in geographically distant natural populations of flies. This may be due to 'infection' with a movable element. In addition, a mutation was found which influences both $s n$ and $c t$ loci and probably depends on the presence of the same element. A correlation between instability in white locus and mog 1 insertion was shown by E.S. Belyaevat.

The localization of the mdg elements in chromosomes appears highly conserved during differentiation as they have been found in the same position in cells of salivary glands and stomach (E.V. Ananiev ${ }^{\dagger}$ ). They are also stable in inbred lines of $D$. melanogaster. The probability of 'jumping' for mdgl and mdg3 was estimated to be of the order of $10^{-5}$.

Drastic changes in $\mathrm{mdgl}$ and $\mathrm{mdg} 3$ localization were found to take place during back selection for high sexual activity among inbred flies characterized by low sexual activity (V.S. Gvozdev'). Such transpositions might facilitate adaptation of the organism to the environment through influence on the expression of other genes.

Mdg elements appear species specific: those of $D$. melanogaster cannot be detected in $D$. virilis and vice versa (M.B. Evgen'ev*). A new mdg family has been cloned by $\mathrm{G}$. N. Yenikolopov from $D$. virilis genome. $D$. littoralis chromosomes included in the genome of $D$. virilis are able to accept $D$. virilis mdg copies without crossing-over.

A.N. Gudkov (Oncological Centre, Moscow) discussed endogenous proretroviruses and their similarity to $\mathrm{mdg}$ elements. Different strains of hen differ strikingly in the number and localization of proviral sequences as studied by Southern blotting analysis. The existence of a natural strain completely lacking RAVo-like sequences was described. Together with the data of Astrin et al. (Cold Spring Harb. Symp. quant. Biol 344; 1105, 1979) this suggests that the presence of proviral sequences is not essential for the organism.

Sequences homologous to the oncogene of mouse Moloney sarcoma virus (putative protooncogene) and to some other sequences of the virus (human C-type proretrovirus) are found in the normal human genome (E.A. Zabarovsky*). The protooncogene-containing sequence has been cloned from the human genome.

D.M. Kramerov* described the properties of two mdg-like elements cloned from the mouse genome selected on the basis of efficient hybridization to long stretches of double-stranded RNA (dsRNA-A). One of these cloned sequences was found to be responsible for the synthesis of intracisternal A-particles. Also, endogeneous $C$-type proviruses were detected among dsRNA-A-hybridizing sequences of mouse genome.

Structural similarities between $\mathrm{mdg}$ elements of Drosophila and endogenous proretroviruses were stressed by Georgiev. $\mathrm{He}$ also discussed a 'promoter' hypothesis of carcinogenesis (see $J$. theor. Biol. $25 ; 473,1969)$ which considers oncogenic transformation to result from a potential cellular oncogene falling under the control of the viral promoter. Mdg elements may provide an important source of such poorly controlled promoters in the course of nonviral oncogenesis. 